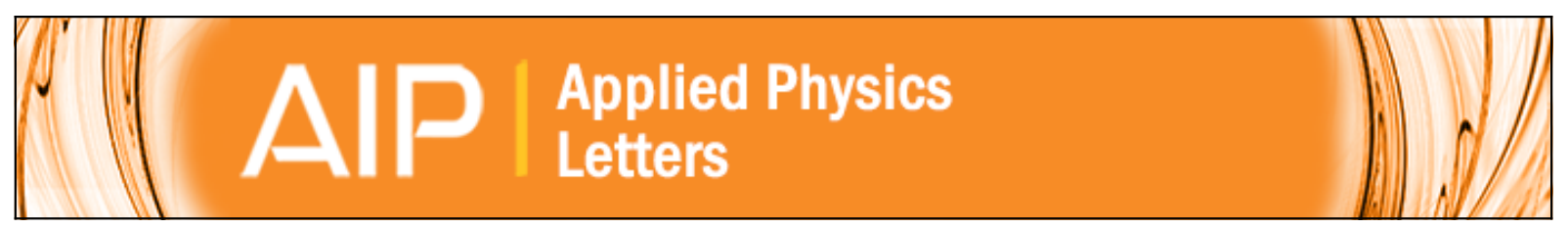

\title{
Magnetoelectric control of spin currents
}

J. E. Gómez, J. M. Vargas, L. Avilés-Félix, and A. Butera

Citation: Applied Physics Letters 108, 242413 (2016); doi: 10.1063/1.4954167

View online: http://dx.doi.org/10.1063/1.4954167

View Table of Contents: http://scitation.aip.org/content/aip/journal/apl/108/24?ver=pdfcov

Published by the AIP Publishing

\section{Articles you may be interested in}

Magneto-electric effects in functionally stepped magnetic nanobilayers on ferroelectric substrates: Observation and theory on the influence of interlayer exchange coupling

J. Appl. Phys. 115, 193909 (2014); 10.1063/1.4878458

Electrically controlled magnetization switching in a multiferroic heterostructure

Appl. Phys. Lett. 97, 052502 (2010); 10.1063/1.3475417

Large converse magnetoelectric coupling in FeCoV/lead zinc niobate-lead titanate heterostructure

Appl. Phys. Lett. 94, 082504 (2009); 10.1063/1.3086879

Electric-field-induced magnetization in $\mathrm{Pb}$ ( $\mathrm{Zr}, \mathrm{Ti}$ ) $\mathrm{O} 3 /$ Terfenol-D composite structures

Appl. Phys. Lett. 88, 182502 (2006); 10.1063/1.2199967

Large high-frequency magnetoelectric response in laminated composites of piezoelectric ceramics, rare-earth iron alloys and polymer

Appl. Phys. Lett. 84, 3516 (2004); 10.1063/1.1739277

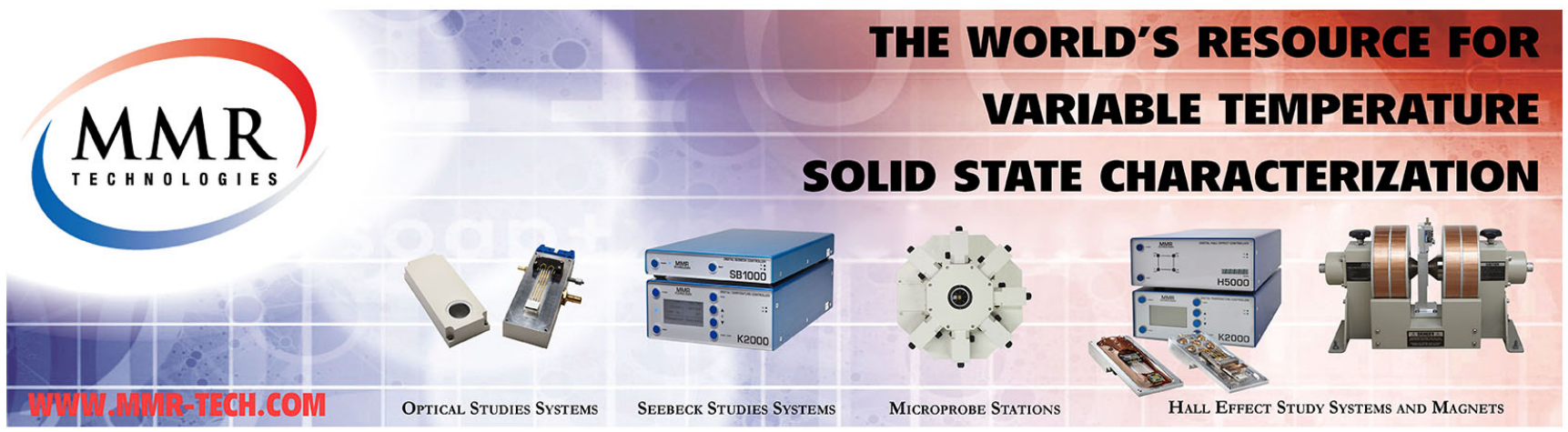




\title{
Magnetoelectric control of spin currents
}

\author{
J. E. Gómez, J. M. Vargas, L. Avilés-Félix, and A. Butera \\ Centro Atómico Bariloche, Instituto de Nanociencia y Nanotecnología (CNEA) and Conicet, 8400 Bariloche, \\ Río Negro, Argentina
}

(Received 4 May 2016; accepted 4 June 2016; published online 15 June 2016)

\begin{abstract}
The ability to control the spin current injection has been explored on a hybrid magnetoelectric system consisting of a (011)-cut ferroelectric lead magnesium niobate-lead titanate (PMNT) single crystal, a ferromagnetic FePt alloy, and a metallic Pt. With this PMNT/FePt/Pt structure we have been able to control the magnetic field position or the microwave excitation frequency at which the spin pumping phenomenon between FePt and Pt occurs. We demonstrate that the magnetoelectric heterostructure operating in the L-T (longitudinal magnetized-transverse polarized) mode couples the PMNT crystal to the magnetostrictive $\mathrm{FePt} / \mathrm{Pt}$ bilayer, displaying a strong magnetoelectric coefficient of $\sim 140 \mathrm{Oe} \mathrm{cm} \mathrm{kV}^{-1}$. Our results show that this mechanism can be effectively exploited as a tunable spin current intensity emitter and open the possibility to create an oscillating or a bistable switch to effectively manipulate spin currents. Published by AIP Publishing.

[http://dx.doi.org/10.1063/1.4954167]
\end{abstract}

The future of spintronic devices relies on the ability to overcome the different challenges linked to this emerging field, in particular, the generation, manipulation, and detection of a spin current. Consequently, over the last decades an enormous effort has been focused on the manipulation of the spin degree of freedom of the charge carriers. ${ }^{1-3}$ In such an effort, the discovery of the spin pumping phenomenon ${ }^{4,5}$ opened a new path to investigate, understand, and potentially design spin based devices. The spin pumping can be thought as a pure spin current appearing in a ferromagnet/normal metal (FM/NM) interface when the ferromagnet is fulfilling the magnetic resonance condition under microwave excitation. The appearance of the spin current is a consequence of an interfacial relaxation process, mediated by the conduction electrons of the normal metal, which contributes to relax the magnetization of the FM to its equilibrium configuration. The spin current is continuously injected and propagates diffusively inside the normal metal volume, perpendicularly to the interface. It is well known that a direct and sensitive detection of spin currents can be done using the inverse spin Hall effect (ISHE). ${ }^{6-8}$ This effect arises in the strong spinorbit coupling of some heavy metals like Pt, which deflects the trajectory of electrons (with opposite spins and propagation velocities) preferably to one edge of the sample generating a measurable voltage, the ISHE signal.

Controlling spin related properties by electronic means is a key step toward future spintronic technologies. Tuning the response using electric $E$-fields is rapid, less noisy, and is compatible with device miniaturization. FePt equiatomic alloy thin films usually grow in a metastable chemically disordered fcc phase (called A1), which presents a saturation magnetization similar to that of the ordered $\mathrm{L} 1_{0}$ phase $\left(M_{s} \sim 1100 \mathrm{emu} / \mathrm{cm}^{3}\right)$, but has considerably smaller anisotropy and coercive fields. ${ }^{9} \mathrm{FePt}$ alloy also displays potentially interesting magnetostrictive properties. ${ }^{9-11}$ The A1 phase, in contrast to the ordered $\mathrm{L} 1_{0}$ phase, has a well defined magnetic resonance absorption line, ${ }^{11}$ making it ideal to be used as a test-bed magnetostrictive film in spin pumping investigations. In previously published results ${ }^{12}$ we have also analyzed the microwave properties of a magnetoelectric (ME) lead magnesium niobate-lead titanate (PMNT)/FePt heterostructure under $E$ - and magnetic $H$-fields tuning control. We found that in a $20 \mathrm{~nm}$ FePt thin film, the magnetoelectric coupling can be higher than $40 \mathrm{Oe} \mathrm{cm} \mathrm{kV}^{-1}$. Based on our recent work on the response of hybrid magnetoelectric systems, we have designed an ISHE tunable system consisting of a substrate/bilayer structure made up of a (011)-cut PMNT single crystal and an FePt/Pt bilayer. In this study we have focused on the capability of this magnetoelectric structure to control the spin pumping by using a static $E$-field. A good agreement with the predictions of both inverse spin Hall effect and magnetoelastic theories has been obtained.

Samples were grown on the polished side of a lead magnesium niobate-lead titanate (011) single crystal with a nominal thickness of $0.05 \mathrm{~cm}$. PMNT is a ferroelectric material that exhibits a giant strain hysteresis controllable with electric fields. ${ }^{13,14}$ In particular, (011)-cut PMNT single crystals display large anisotropic in-plane piezoelectric coefficients with a negative $d_{31}$ around $-1200 \mathrm{pC} \mathrm{N} \mathrm{N}^{-1}$ and a positive $d_{32}$ of $\sim 400 \mathrm{pC} \mathrm{N}^{-1} \cdot{ }^{14,15}$ Using dc magnetron sputtering we deposited $50 \mathrm{~nm}$ of $\mathrm{Ag}$ on the unpolished side as the bottom electrode. On the polished side of the crystal we deposited the two thin films consisting of $20 \mathrm{~nm}$ of FePt covered by $10 \mathrm{~nm}$ of Pt, with lateral size of $4 \mathrm{~mm} \times 1 \mathrm{~mm}$. A sketch of the sample is depicted in Fig. 1.

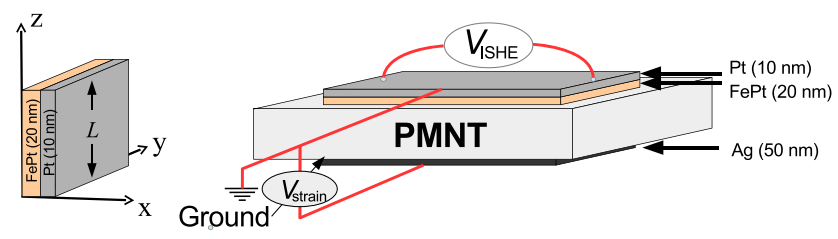

FIG. 1. Sketch of the magnetoelectric structure consisting of an $\mathrm{FePt}(20 \mathrm{~nm}) / \mathrm{Pt}(10 \mathrm{~nm})$ bilayer grown on a (011) PMNT crystal slab with a bottom $50 \mathrm{~nm} \mathrm{Ag} \mathrm{electrode.} \mathrm{In} \mathrm{the} \mathrm{inset} \mathrm{we} \mathrm{show} \mathrm{the} \mathrm{coordinate} \mathrm{reference}$ system chosen to describe the FMR and the ISHE signal measurements. 
Spin pumping through the metallic interface was induced by driving the FePt layer to the ferromagnetic resonance (FMR) condition. The experiment was performed at room temperature in a commercial Bruker ESP300 spectrometer at a microwave frequency of $9.78 \mathrm{GHz}$ (X bandTE102 rectangular cavity). The ISHE signal was acquired by measuring the voltage between the edges of the sample (see Fig. 1) as described in previously published works. ${ }^{6,16}$ Measurements were made with the magnetic field applied parallel to the film plane along the $y$ direction, with the microwave excitation field parallel to $z$. Due to the hysteretic behavior of the PMNT, it is necessary to follow a systematic protocol for the application of the $E$-field in order to get repetitive and reliable results. Before starting the measurements the PMNT was cycled by applying alternatively electric fields of $\pm 4 \mathrm{kV} / \mathrm{cm}$, and then $E=-4 \mathrm{kV} / \mathrm{cm}$ was used as the starting point for the experiments.

In Fig. 2(a) we present typical X-Band data for the FMR absorption line and the ISHE signal. A single line is observed in both cases. The FMR absorption spectra show a derivative Lorentzian lineshape due to the modulation field used for detection, while ISHE signals are Lorentzian, with a maximum voltage, $V_{\text {ISHE }}$, occurring at a field $H_{\text {ISHE }}$ coincident with the zero crossing of the FMR absorption. The small absorption observed at 1640 Oe in Fig. 2(a) is due to the pertinax sample holder.

Figure 2(b) shows the trend followed by the ISHE signal at different $E$-fields. It is observed that $H_{\text {ISHE }}$ shifts to larger fields when the absolute value of the $E$-field is increased. The application of a voltage between both electrodes produces a strain on the PMNT which in turn causes a mechanical elastic deformation on the FePt/Pt bilayer. Due to the magnetostrictive properties of the FePt an additional strain anisotropy field is induced, which modifies the resonance field position. A detail of the response of $H_{\text {ISHE }}$ and $V_{\text {ISHE }}$

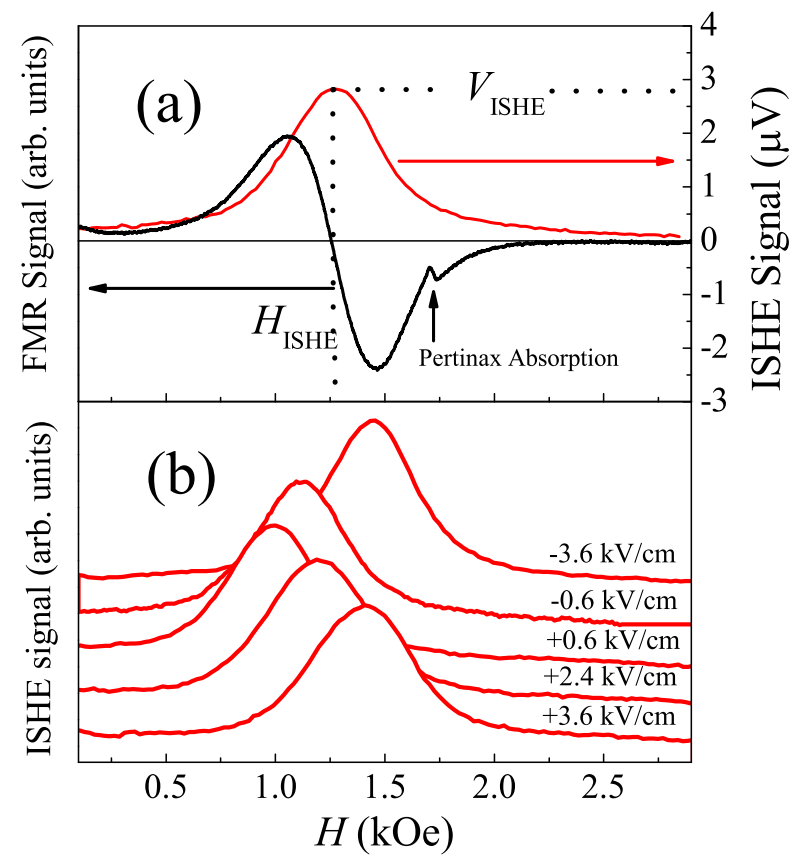

FIG. 2. (a) Typical ISHE signal and FMR spectrum of the PMNT/FePt/Pt system for $E=2.6 \mathrm{kV} / \mathrm{cm}$. (b) Evolution of the ISHE signal for different $E$-field values. against the $E$-field is shown in Fig. 3 and the inset, respectively. The already mentioned protocol was used to obtain the ascending branch from $-4 \mathrm{kV} / \mathrm{cm}$ to $+4 \mathrm{kV} / \mathrm{cm}$ (full square symbols) and then the descending from 4 to $-4 \mathrm{kV} / \mathrm{cm}$ (open circle symbols). The variation of $H_{\mathrm{ISHE}}$ vs. $E$-field shows a non-reversible butterfly-like hysteretic shape that follows closely the $E$-field induced strain in the ferroelectric crystal. ${ }^{17-19}$ The maximum of the ISHE signal is weakly dependent on $E$ (see the inset of the Fig. 3) with an average $V_{\text {ISHE }}$ $\sim 2.8 \pm 0.3 \mu \mathrm{V}$.

As the ISHE signal is a direct measurement of the spin current pumped during the FMR experiment, the behavior shown in Fig. 3 is a strong evidence that the spin current emission can be controlled by using $E$-fields. These results proved an alternative way to tune the magnetic field at which the spin current is established. With the aim to quantitatively understand the control of the spin current emission by the $E$-field, the effects of the mechanical strain on the PMNT crystal must be considered simultaneously in the ISHE and magnetoelastic theories. The strain as a function of $E$ in the two in-plane principal axes of a (011)-cut PMNT single crystal has been carefully studied by several authors. ${ }^{13-15}$ It is thus important to remark the complex non-reversible anisotropic behavior of ferroelectric materials. The strain occurring in the crystal is converted in an asymmetric stress on the bilayer, with $\sigma_{y} \neq \sigma_{z} \neq 0$ and $\sigma_{x}=0$ (the $\mathrm{FePt} / \mathrm{Pt}$ is unstressed in the normal direction) where $\sigma_{x, y, z}$ refers to the stress components. The relevant contributions to the free energy can then be written as

$$
\begin{aligned}
E_{F}= & -\mathbf{H} \cdot \mathbf{M}+2 \pi M^{2} \sin ^{2} \theta \cos ^{2} \phi \\
& -\frac{3}{2} \lambda\left(\sigma_{y} \sin ^{2} \theta \sin ^{2} \phi+\sigma_{z} \cos ^{2} \theta\right),
\end{aligned}
$$

where $\theta$ and $\phi$ are the azimuth and polar angles of the magnetization $\mathbf{M}$ in the reference system of the sample (see Fig. 1).

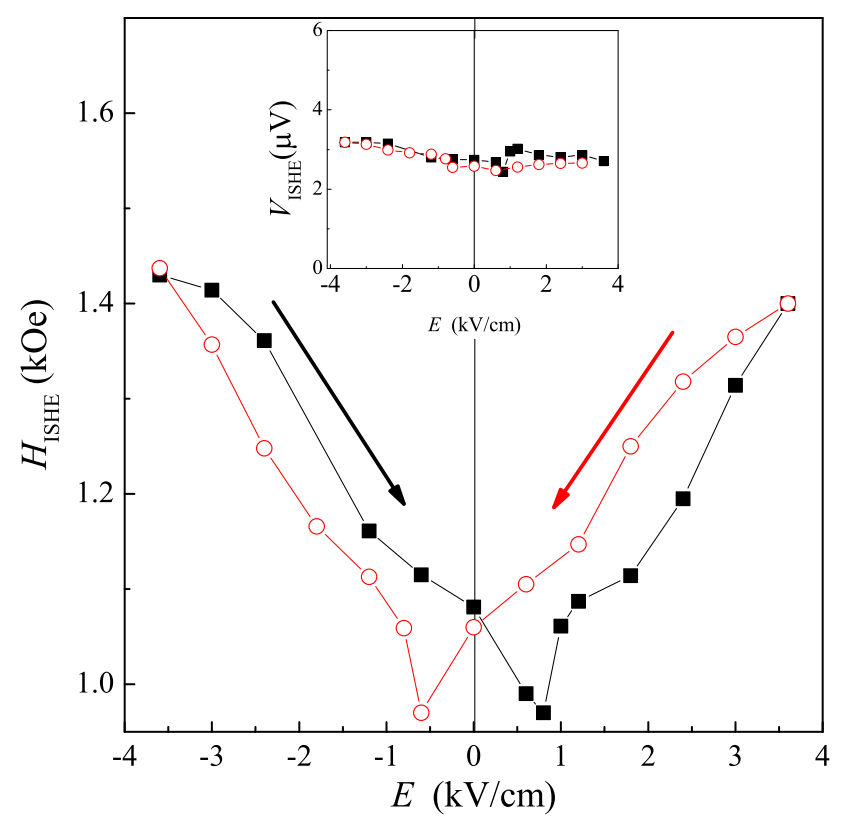

FIG. 3. Dependence of the magnetic field where the maximum ISHE signal is observed $\left(H_{\text {ISHE }}\right)$ as a function of the $E$-field. The inset shows that the voltage values of $V_{\text {ISHE }}$ are almost independent on the $E$-field. 
The first and second terms of Eq. (1) correspond to the Zeeman and shape anisotropy energies while the last term is the magnetoelastic energy. ${ }^{20}$ Note that a charge mediated surface anisotropy term is often considered in the description of the free energy, especially in the case of very thin films. ${ }^{18}$ This anisotropy depends on the polarization state of the PMNT causing an asymmetry in the resonance field position when it is plotted as a function of the E-field. As the asymmetry observed in Fig. 3 is negligibly small, we did not consider this charge mediated anisotropy field in Eq. (1).

The Smit-Beljers formalism is generally used to connect the microwave frequency excitation with the magnetic and anisotropy fields. ${ }^{21}$ The corresponding dispersion relation takes the form of Eq. (2) when the external magnetic field is applied along the $y$ direction (the equilibrium angles of the magnetization are $\phi=\theta=\pi / 2$ )

$$
\left(\frac{\omega}{\gamma}\right)^{2}=\left(H+H_{1}\right)\left(H+H_{\text {eff }}+H_{2}\right),
$$

with $\omega / 2 \pi$ the microwave excitation frequency, $\gamma=g \mu_{B} / \hbar$ the gyromagnetic ratio, and $\lambda$ the saturation magnetostriction coefficient of FePt. The magnetoelastic and the shape anisotropy fields of Eq. (2) are defined as $H_{1}=\frac{3 \lambda}{M}\left(\sigma_{y}-\sigma_{z}\right)$, $H_{2}=\frac{3 \lambda}{M} \sigma_{y}$, and $H_{\text {eff }}=4 \pi M$.

As already mentioned, PMNT crystals have $d_{31}$ and $d_{32}$ coefficients of opposite sign, which gives $\sigma_{y}$ and $\sigma_{z}$ stresses of different sign and consequently $\left|H_{1}\right|>\left|H_{2}\right|$. Additionally, an estimation of these magnetoelastic anisotropy fields indicates that they are both much smaller than the shape anisotropy field, i.e., $\left|H_{1}\right|,\left|H_{2}\right| \ll H_{\text {eff }}$. When this condition holds, the dispersion relation can be approximated by

$$
\left(\frac{\omega}{\gamma}\right)^{2} \sim\left(H+H_{1}\right)\left(H+H_{\mathrm{eff}}\right) .
$$

Eq. (3) shows that the effective magnetoelastic field $H_{1}$ can be associated to the changes in the magnetic field position at which $V_{\text {ISHE }}$ occurs. From the experimental results presented in Fig. 3 we have found that the total variation of $H_{1}$ in the spanned range of $E$-fields is $\Delta H_{1} \sim 500 \mathrm{Oe}$. Considering and average slope in the ascending and descending branches of Fig. 3 (due to the hysteresis in the ferroelectric crystal) we can estimate an ME coefficient of $\sim 140$ Oe $\mathrm{cm} / \mathrm{kV}$.

This ME coupling value can be compared with values reported by Liu et al. in $\mathrm{Fe}_{3} \mathrm{O}_{4} /(011)$-cut PMNT. ${ }^{19}$ They observed an ME coefficient of $67 \mathrm{Oe} \mathrm{cm} \mathrm{kV}^{-1}$. An enhanced value was obtained using a single crystal slab of Lead Zinc Niobate-Lead Titanate (PZNT) in a $\mathrm{Fe}_{3} \mathrm{O}_{4} / \mathrm{PZNT}$ heterostructure, with an $\mathrm{ME}$ value of $108 \mathrm{Oe} \mathrm{cm} \mathrm{kV}^{-1} \cdot{ }^{19}$ In addition, our value can be compared with the FeGaB/PZNT heterostructure, which shows an average $\mathrm{ME}$ value of $94 \mathrm{Oe} \mathrm{cm} \mathrm{kV}^{-1}$.

On the other hand, in order to analyze $V_{\text {ISHE }}$ as a function of the $E$-field, it is necessary to explore the effect of the magnetoelastic anisotropy fields on the spin current pumped during the FMR experiment. It is important to remember that the ISHE signal comes from the ability of the Pt layer to convert the spin current into a measurable voltage. ${ }^{5}$ The magnetization precessing in the ferromagnetic film loses angular momentum generating an interfacial (FePt/Pt) spin current, given by the following equation:

$$
J_{s}^{0}=\frac{\omega}{2 \pi} g_{\text {eff }}^{\uparrow \downarrow} \frac{\hbar}{4 \pi}\left|\int_{0}^{\frac{2 \pi}{\omega}}\left(\mathbf{m} \times \frac{d \mathbf{m}}{d t}\right) d t\right|
$$

where $g_{\text {eff }}^{\uparrow \downarrow}$ is the effective spin mixing conductance that quantifies the efficiency of the FM layer to transfer angular momentum across the FM/NM interface. The spin current propagates diffusively across the thickness of the Pt film and vanishes at the opposite interface (Pt/air). ${ }^{4}$ The magnetoelastic anisotropy fields modify the ISHE signal because of the implicit dependence contained in Eq. (4). A solution of this equation is quite complex but when the resonance condition is fulfilled $\left(H=H_{\text {ISHE }}\right)$ it takes a simpler form given by the following expression:

$$
J_{s}^{0}=\gamma \frac{\hbar}{4 \pi} g_{\mathrm{eff}}^{\uparrow \downarrow}\left(\frac{h_{\mu \omega}}{\alpha}\right)^{2} \frac{H_{\mathrm{ISHE}}+H_{\mathrm{eff}}+H_{2}}{\left[2 H_{\mathrm{ISHE}}+H_{\mathrm{eff}}+H_{1}+H_{2}\right]^{2}},
$$

where $h_{\mu \omega}$ is the amplitude of the microwave magnetic field and $\alpha$ is the Gilbert damping parameter. As already mentioned, the shape anisotropy field is much larger than other fields and, as can be inferred from Eq. (5), the variation of the other fields produces very small changes in $J_{s}^{0}$. This dependence explains the relatively constant value of $V_{\text {ISHE }}$ as a function of $E$-field observed in the inset of Fig. 3.

A remarkable result is found when the $E$-field is used to detune the FMR condition. If the external $H$ - and $E$-fields are initially chosen in order to maximize the emission of the spin current, it is then possible to modify $E$ in order to detune the spin pumping condition. When the $H$-field is kept constant, the spin current intensity can be controlled exclusively by the $E$ magnitude. The behavior is shown in Fig. 4, where $V_{\text {ISHE }}$ as a function of the $H$-field is plotted for different values of $E$. If $H$ is maintained fixed, for example, at 1430 Oe as indicated by a vertical line in the figure, a strong $E$-field dependence of the ISHE signal is found. The results obtained from this experiment show explicitly that a precise

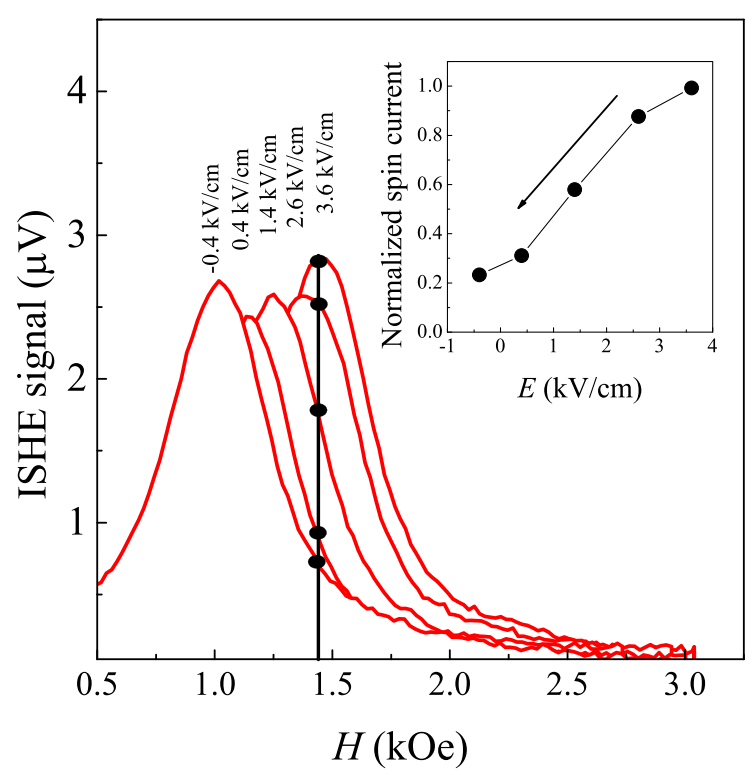

FIG. 4. ISHE signal as a function of $H$-field measured at different $E$-fields. The inset shows the evolution of the spin current intensity as a function of $E$ when the $H$-field is kept fixed at the value indicated by a vertical line. 
control of the spin current intensity can be obtained by changing the external $E$-field. As can be seen in the inset of Fig. 4, when the $E$-field is changed from $3.6 \mathrm{kV} / \mathrm{cm}$ to $-0.4 \mathrm{kV} / \mathrm{cm}$ the spin current intensity is reduced to $20 \%$ of the initial value. By properly defining threshold limits, this behavior can also be used to build a voltage controlled ON/ OFF spin current device.

Because of the hysteretic behavior of the PMNT crystal, some caution must be taken in the interpretation of the results. The precise control of the spin current depends on the history of the experiment, and is not reversible for $E$-fields within the hysteresis region. However, if a protocol is followed the results are reproducible. The strain-field response of PMNT crystals is nonhysteretic only in the region of $|E| \geqslant 10 \mathrm{kV} / \mathrm{cm}$. If a reversible response is desired, for example, when trying to obtain an oscillating spin current by driving the PMNT with an oscillating voltage, it would be necessary to apply a bias voltage to shift the operating region to the reversible part of the hysteresis loop. The application of relatively large electric fields implies the possibility of a dielectric breakdown failure. The design of structures that can work in the nonhysteretic region of the ferroelectric material is an open challenge for future investigations in optimized hybrid magnetoelectric structures.

The reduction of the bias $H$-field could be attained by reducing the microwave excitation frequency or by inducing an uniaxial anisotropy in the fabrication process of the sample. Both parameters can be modified in order to tune the resonance condition at a lower or even zero applied magnetic field. In this condition the possibility of the formation of magnetic domains makes the analysis more complex and extra care must be taken in the interpretation of the experimental data.

In summary, we have demonstrated that an $E$-field can be used to modify the magnetic field position at which the spin pumping phenomenon occurs in a magnetoelectric structure $(\mathrm{PMNT} / \mathrm{FePt} / \mathrm{Pt})$. Our results also show the possibility of controlling the pure spin current intensity by using an electric field. Additionally, we have opened the possibility to create an oscillating spin current device or an ON/OFF switch with very good control of the spin current. All these results, obtained from the $E$-field control of the ISHE signal, are promising for the development of spin based devices and can lead to substantial advances in hybrid spintronic devices.

This work was supported in part by Conicet (Grant No. PIP 112-201101-00482), ANPCyT (Grant Nos. PICT 20132363 and PICT 2013-0401), and U.N. Cuyo (Grant No. 06/ C421), all from Argentina. The fruitful discussions with Dr. Carlos González Oliver and the signal conditioning advice of Engineer Neil P. Albaugh are greatly acknowledged.

${ }^{1}$ K. Uchida, S. Takahashi, K. Harii, J. Ieda, W. Koshibae, K. Ando, S. Maekawa, and E. Saitoh, Nature 455, 778 (2008).

${ }^{2}$ Y. Kajiwara, K. Harii, S. Takahashi, J. Ohe, K. Uchida, M. Mizuguchi, H. Umezawa, H. Kawai, K. Ando, K. Takanashi et al., Nature 464, 262 (2010).

${ }^{3}$ J. Sinova, D. Culcer, Q. Niu, N. A. Sinitsyn, T. Jungwirth, and A. H. MacDonald, Phys. Rev. Lett. 92, 126603 (2004).

${ }^{4}$ Y. Tserkovnyak, A. Brataas, and G. E. W. Bauer, Phys. Rev. Lett. 88, 117601 (2002).

${ }^{5}$ E. Saitoh, M. Ueda, H. Miyajima, and G. Tatara, Appl. Phys. Lett. 88, 182509 (2006).

${ }^{6}$ J. E. Gómez, B. Zerai Tedlla, N. R. Álvarez, G. Alejandro, E. Goovaerts, and A. Butera, Phys. Rev. B 90, 184401 (2014).

${ }^{7}$ O. Mosendz, J. E. Pearson, F. Y. Fradin, G. E. W. Bauer, S. D. Bader, and A. Hoffmann, Phys. Rev. Lett. 104, 046601 (2010).

${ }^{8}$ C. Hahn, G. de Loubens, O. Klein, M. Viret, V. V. Naletov, and J. Ben Youssef, Phys. Rev. B 87, 174417 (2013).

${ }^{9}$ V. Mansilla, J. Gómez, and A. Butera, IEEE Trans. Magn. 44, 2883 (2008).

${ }^{10}$ N. R. Álvarez, J. E. Gómez, A. E. Moya Riffo, M. A. Vicente Álvarez, and A. Butera, J. Appl. Phys. 119, 083906 (2016).

${ }^{11}$ N. Álvarez, G. Alejandro, J. Gómez, E. Goovaerts, and A. Butera, J. Phys. D: Appl. Phys. 46, 505001 (2013).

${ }^{12}$ J. Vargas and J. Gómez, APL Mater. 2, 106105 (2014).

${ }^{13}$ T. Wu, P. Zhao, M. Bao, A. Bur, J. L. Hockel, K. Wong, K. P. Mohanchandra, C. S. Lynch, and G. P. Carman, J. Appl. Phys. 109, 124101 (2011).

${ }^{14}$ P. Han, W. Yan, J. Tian, X. Huang, and H. Pan, Appl. Phys. Lett. 86, 052902 (2005).

${ }^{15}$ I. A. Ivan, M. Rakotondrabe, J. Agnus, R. Bourquin, N. Chaillet, P. Lutz, J. C. Poncot, R. Duffait, and O. Bauer, Rev. Adv. Mater. Sci. 24, 1 (2010).

${ }^{16}$ J. E. Gómez, M. Guillén, A. Butera, and N. P. Albaugh, Rev. Sci. Instrum. 87, 024705 (2016).

${ }^{17}$ Z. Zhou, S. Zhao, Y. Gao, X. Wang, T. Nan, N. X. Sun, X. Yang, and M. Liu, Sci. Rep. 6, 20450 (2016).

${ }^{18}$ T. Nan, Z. Zhou, M. Liu, X. Yang, Y. Gao, B. A. Assaf, H. Lin, S. Velu, X. Wang, H. Luo et al., Sci. Rep. 4, 3688 (2014).

${ }^{19}$ M. Liu, O. Obi, J. Lou, Y. Chen, Z. Cai, S. Stoute, M. Espanol, M. Lew, X. Situ, K. S. Ziemer, V. G. Harris, and N. X. Sun, Adv. Funct. Mater. 19, 1826 (2009).

${ }^{20}$ S. Chikazumi, Physics of Magnetism (Krieger Publishing, Florida, 1964), p. 182.

${ }^{21}$ J. Smit and H. G. Beljers, Philips Res. Rep. 10, 113 (1955). 